\title{
OPEN Molecular-Based Score inspired on metabolic signature improves prognostic stratification for myelodysplastic syndrome
}

\author{
Juan L. Coelho-Silva ${ }^{1,2}$, Douglas R. A. Silveira ${ }^{3,4}$, Diego A. Pereira-Martins ${ }^{2,5}$, \\ Cesar A. O. Rojas ${ }^{2}$, Antonio R. Lucena-Araujo6, Eduardo M. Rego ${ }^{2,3}$, João A. Machado-Neto7, \\ Israel Bendit ${ }^{3}$, Vanderson Rocha ${ }^{3} \&$ Fabiola Traina ${ }^{1,2}$
}

Deregulated cellular energetics is formally incorporated as an emerging hallmark of cancer, however little is known about its processes in myelodysplastic syndromes (MDS). Using transcriptomic data of CD34+ cells from 159 MDS patients and 17 healthy donors, we selected 37 genes involved in cellular energetics and interrogated about its clinical and prognostic functions. Based on the low expression of ACLY, ANPEP, and PANK1, as well as high expression of PKM and SLC25A5, we constructed our Molecular-Based Score (MBS), that efficiently discriminated patients at three risks groups: favourable risk ( $n=28$; 3-year overall survival (OS): $100 \%)$; intermediate $(n=60 ; 76 \%[62-93 \%])$ and adverse $(n=71 ; 35 \%[17-61 \%])$. Adverse MBS risk was independently associated with inferior OS (HR $=10.1$ [95\% Cl 1.26-81]; $P=0.029$ ) in multivariable analysis using age, gender and the revised international prognostic score system as confounders. Transcriptional signature revealed that Favourable- and intermediate-risk patients presented enriched molecular programs related to mature myeloid progenitors, cell cycle progression, and oxidative phosphorylation, indicating that this cells differs in their origin, metabolic state, and cell cycle regulation, in comparison to the adverse-risk. Our study provides the first evidence that cellular energetics is transcriptionally deregulated in MDS CD34+ cells and establishes a new useful prognostic score based on the expression of five genes.

Myelodysplastic syndromes (MDS) are a heterogeneous group of clonal myeloid neoplasms, which are characterized by bone marrow failure, abnormal cell morphology, and increased risk for evolution to acute myeloid leukaemia ${ }^{1}$. The recent efforts to uncover the molecular heterogeneity of MDS, mainly by new sequencing technologies, has continually allowed the comprehensive identification of driver mutations or altered gene expression recurrently found in a recognizable fraction of patients $s^{2,3}$. Deregulated gene expression is prognostically useful in haematological neoplasms, but still underexplored in $\mathrm{MDS}^{4,5}$. Moreover, very few data, if any, are available considering deregulated gene expression processes of MDS-initiating cell.

Cancer cells preferentially upregulates glucose uptake and glycolysis to give rise to increased yield of intermediate glycolytic metabolites, and, as consequence, glycolysis is uncoupled from the mitochondrial tricarboxylic acid (TCA) cycle and oxidative phosphorylation (OXPHOS) in cancer cells ${ }^{6,7}$. This effect, also known as Warburg effect, results in reduced mitochondrial oxidative metabolism ${ }^{6,8,9}$, and deregulated cellular energetics is formally incorporated as an emerging hallmark of cancer ${ }^{10,11}$. Yet, besides the concept of how glucose metabolism influences cellular functions, studies still necessary in order to properly define if the up-regulation of anaerobic glycolysis is a true cancer cell-specific deviation or related to normal stem/progenitor cell maintenance and self-renewal mechanisms ${ }^{12}$.

\footnotetext{
${ }^{1}$ Department of Medical Imaging, Haematology, and Oncology, Ribeirão Preto Medical School, University of São Paulo, Ribeirão Preto, SP, Brazil. ${ }^{2}$ Center for Cell-Based Therapy, São Paulo Research Foundation, Ribeirão Preto, SP, Brazil. ${ }^{3}$ Hematology Division, LIM31, Faculdade de Medicina, University of São Paulo, São Paulo, SP, Brazil. ${ }^{4}$ Department of Haematology, AC Camargo Cancer Centre, Sao Paulo, Brazil. ${ }^{5}$ Department of Internal Medicine, Ribeirão Preto Medical School, University of São Paulo, Ribeirão Preto, SP, Brazil. ${ }^{6}$ Department of Genetics, Federal University of Pernambuco, Recife, Brazil. ${ }^{7}$ Department of Pharmacology, Institute of Biomedical Sciences, University of São Paulo, São Paulo, SP, Brazil. ${ }^{\circledR}$ email: ftraina@fmrp.usp.br
} 


\begin{tabular}{|l|l|l|l|}
\hline \multicolumn{4}{|l|}{ Cellular energetics-related genes } \\
\hline$A B A T$ & GOT2 & IDH3A & PFKL \\
\hline$A C L Y$ & GPX1 & IDH3B & PFKP \\
\hline ANPEP & GSR & IDH3G & PKM \\
\hline CAT & GSS & LDHA & PKMYT1 \\
\hline CS & GSTM1 & LDHB & SCD \\
\hline DPYPD & HK1 & MDH2 & SDHA \\
\hline ERCC2 & HK2 & ME1 & SLC2A5 \\
\hline FASN & IDH1 & OGDH & SLC25A5 \\
\hline GAD1 & IDH2 & PANK1 & TALDO1 \\
\hline GGCT & & & \\
\hline
\end{tabular}

Table 1. Cellular energetics-related genes selected for the study.

The in-depth evaluation of MDS-initiating metabolism provided by Stevens et al. demonstrated that the CD123 + hematopoietic progenitor compartment is the clonal reservoir for MDS maintenance and evolution ${ }^{13}$. This CD123 + stem cells have distinctive metabolic properties, and the upregulation of protein synthesis, RNA translation, and increased oxidative phosphorylation were directly linked to MDS stem cell self-renewal and survival $^{13}$. Mutations in the SF3B1 gene, represents a subset of MDS with favourable prognosis, results in reprogramming of mitochondrial metabolism related to decreased cellular respiration capacity in a process mediated by the mis-splicing of and downregulation of UQCC $1^{14}$. Therefore, identification of metabolic vulnerabilities in MDS-initiating cells represents a promising strategy to better understand the pathophysiology and propose new therapeutical vulnerabilities for MDS patients.

Our rationale was to design a prognostic score interrogating the clinical and prognostic importance of transcriptionally-regulated enzymes involved in cellular energetics mechanisms of glycolysis, tricarboxylic acid cycle, and oxidative phosphorylation, and to depict the molecular process mediated by our proposed score.

\section{Results}

CD34+ cells from MDS show differential gene expression for cellular energetics-related genes. To examine the differential expression of cellular energetics-related genes, we selected 37 genes (Table 1) and normalize their expression values from microarray data for GSE58831 cohort ${ }^{15}$. The cohort was composed by 159 MDS patients and 17 healthy donors. Nineteen of pre-selected genes were differentially expressed between CD34+ cells from MDS patients and healthy donors (6 downregulated and 13 upregulated; Fig. 1, all $P<0.05)$.

Molecular-Based Score efficiently discriminates MDS patients at differential risk and is associated with clinical and molecular characteristics. To interrogate the prognostic capacity for each selected gene, we dichotomized the gene expression in high- or low-expression according to their receiving operating characteristics (ROC) curve and the C-index. Fifteen genes were associated with prognosis in a univariate analysis, while multivariate analyses identified expression of 5 genes as independent prognostic factors: $A C L Y$ (HR: 0.48; 95\% CI 0.24-0.96; $P=0.04$ ), ANPEP (HR: 2.16; 95\% CI 1.08-4.31; $P=0.02$ ), PANK1 (HR: 0.43; 95\% CI 0.19-0.98; $P=0.04$ ), PKM (HR: 2.01; 95\% CI 1.02-3.93; $P=0.04$ ), and SLC25A5 (HR: 0.49; 95\% CI $0.27-0.99 ; P=0.05$ ) (Table 2). The molecular-Based Score (MBS) was calculated by summing 1 for every gene as a risk factor. The MBS varied from 0 to 5 and was stratified as: MBS Favourable-Risk $=0$ (MBS-FR; $18 \%$ [28/159]); MBS Intermediate-Risk = 1 (MBS-IR; 38\% [60/159]) and Adverse-Risk: $\geq 2$ (MBS-AR; 44\% [71/159]).

Molecular-Based Score efficiently discriminated patients at different risks groups: MBS-FR (3-year overall survival (OS): 100\%; median time [MT]: not reached); MBS-IR (3-year OS: 76\% [95\% CI 62-93\%]; MT: 67.6 months [95\% CI 48.3-86.8]) and MBS-AR (3-year OS: 35\% [95\% CI 17-61\%]; MT: 31.7 months [95\% CI 21.2-42.1]) (Fig. 2A,B). The univariate HRs for IR versus FR and AR versus IR were 8.99 (95\% CI 1.19-68.1; $P=0.02$ ) and 20.1 (95\% CI 0.2.71-149; $P=0.003$ ), respectively (Supplemental Fig. 1). After multivariate adjust, MBS-AR was the most significant covariate as measured by the Wald chi-square statistic and was independently associated to inferior OS ( $\mathrm{HR}=10.1$ [95\% CI 1.26-81]; $P=0.029)$ (Fig. 2C,D). We also identified increased age as an independent prognostic covariate in our model $(\mathrm{HR}=1.03$ [95\% CI 1-1.87]; $P=0.034)$, representing an increment of $3 \%$ of risk of death by year of age at diagnosis (Fig. 2D).

Patients classified as adverse by MBS had significantly decreased platelets counts (median for FR: $250 \times 10^{3} / \mu \mathrm{L}$; IR: $157 \times 10^{3} / \mu \mathrm{L}$ and AR: $\left.109 \times 10^{3} / \mu \mathrm{L} ; P=0.001\right)$ and absolute neutrophil counts (FR: $2.5 \times 10^{3} / \mu \mathrm{L} ; \mathrm{IR}: 2.3 \times 10^{3} /$ $\mu \mathrm{L}$ and AR: $1.3 \times 10^{3} / \mu \mathrm{L} ; P=0.003$ ), while presented higher percentages of bone marrow blasts (FR: $2.5 \%$; IR: $3 \%$ and AR: $8.5 \%$; $P<0.001)$. MBS risk categories were differently distributed across World Health Organization (WHO) MDS entities and IPSS-R classification (both $P<0.001$ ). According to recurrently mutated genes, MBS-AR showed lower frequency of mutations in SF3B1 (FR:50\%; IR: 32\% and AR: 15\%; $P<0.001$ ), and higher frequency of mutations in RUNX1 (FR: 0; IR: 2\% and AR: 13\%; $P=0.03$ ) (Table 3). Collectively, these data suggest a link between MBS and pathophysiology of MDS. MBS Receiving-operating characteristics concordance statistic (ROC C-statistic) was 0.70 (95\% CI 0.62-0.78; Table 4), representing a $20 \%$ improvement in OS prediction when compared with IPSS-R ( $\triangle$-AUC, $0.13 ; 95 \%$ CI $0.02-0.22 ; P=0.01)$. According to IPSS-R risk stratification, MBS 
A
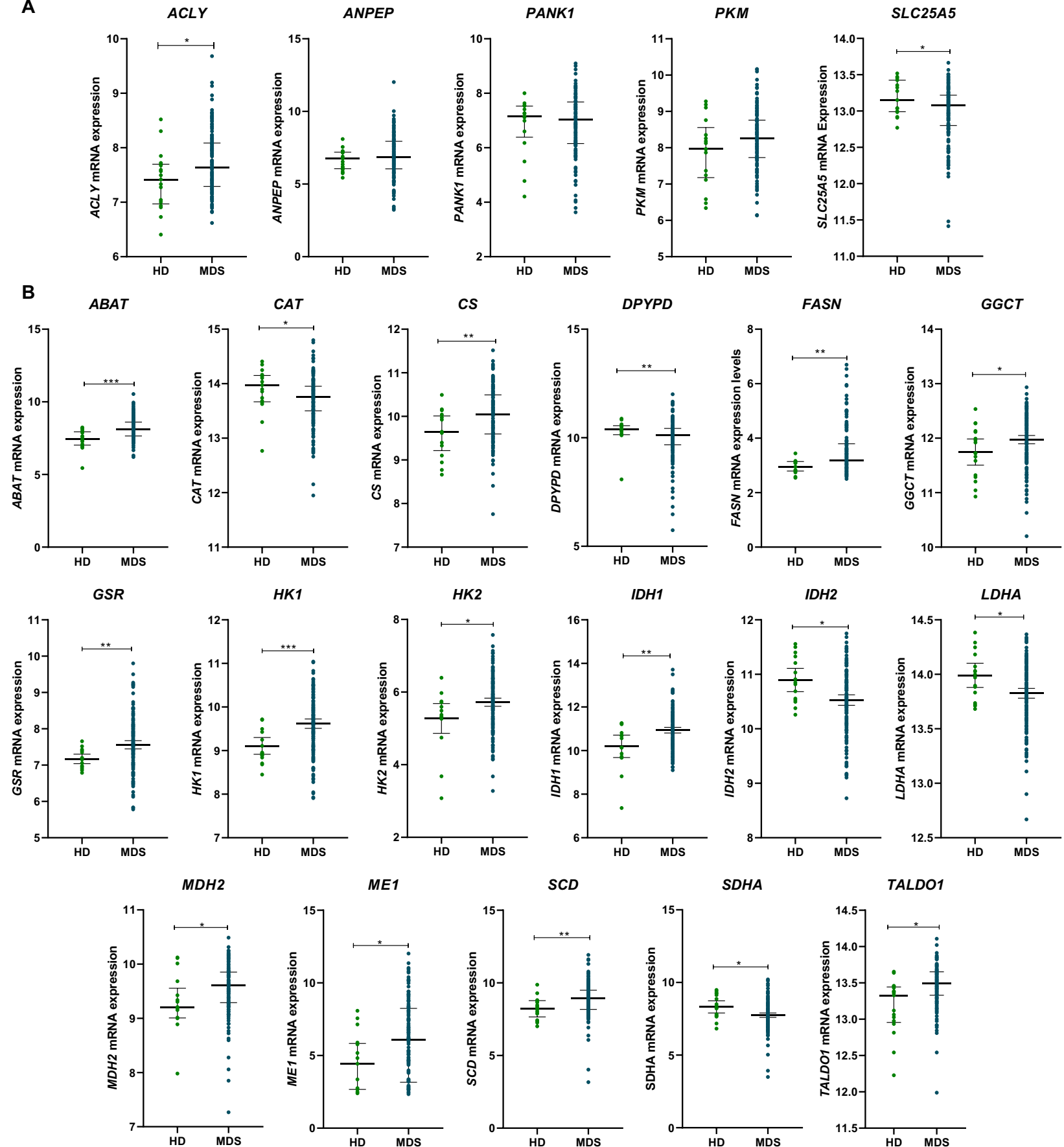

Figure 1. Gene expression from glycolysis and tricarboxylic acid cycle elements in CD34+ cells from healthy donors (HD) and myelodysplastic syndromes patients (MDS). A microarray-based gene expression analysis of selected genes for $17 \mathrm{HD}$ and 159 MDS patients for selected genes used in Molecular Based Score (MBS) (A) and for genes differentially expressed between HD and MDS (B). Horizontal lines indicate medians and the $P$ values are indicated. Notes: ${ }^{\star} P<0.05,{ }^{* *} P<0.01,{ }^{* *} P<0.001$; Mann-Whitney test.

retained its prognostic prediction function when analysed in IPSS-R very-low- and low-risk patients (Fig. 3A) and was widely distributed across all risk categories (Fig. 3B). For non-low IPSS-R patients (i.e., intermediate, high, and very-high), MBS-favourable patients presented a distinctive superior outcome (Supplemental Fig. 3). Of note, none of favourable MBS patients classified as non-low IPSS-R deceased, while 4 of 6 low risk IPSS-R classified as adverse by MBS died with median survival of 18.4 months (Supplemental Table 3). 


\begin{tabular}{|c|c|c|c|c|c|c|}
\hline \multirow[b]{2}{*}{ Target } & \multicolumn{3}{|c|}{ Univariate analysis } & \multicolumn{3}{|c|}{ Multivariate analysis } \\
\hline & Hazard Ratio $^{1}$ & $95 \% \mathrm{CI}$ & $P$ & Hazard Ratio $^{1}$ & $95 \% \mathrm{CI}$ & $P$ \\
\hline GAD1 & 0.34 & $0.17-0.66$ & 0.001 & 0.73 & \begin{tabular}{|l|}
$0.3-1.73$ \\
\end{tabular} & 0.47 \\
\hline ANPEP & 2.73 & $1.44-5.17$ & 0.002 & 2.16 & $1.08-4.31$ & 0.02 \\
\hline$A C L Y$ & 0.38 & $0.21-0.72$ & 0.002 & 0.48 & $0.24-0.96$ & 0.02 \\
\hline$D P Y P D$ & 3.19 & $1.33-7.65$ & 0.008 & 2.22 & \begin{tabular}{|l|}
$0.9-5.48$ \\
\end{tabular} & 0.08 \\
\hline MDH2 & 0.43 & $0.23-0.81$ & 0.009 & 0.6 & $0.31-1.21$ & 0.15 \\
\hline SLC25A5 & 0.44 & $0.24-0.83$ & 0.01 & 0.49 & $0.27-0.99$ & 0.04 \\
\hline GOT2 & 0.37 & $0.17-0.80$ & 0.01 & 0.58 & $0.24-1.36$ & 0.21 \\
\hline$P K M$ & 2.24 & $1.18-4.23$ & 0.01 & 2.01 & $1.02-3.93$ & 0.04 \\
\hline SLC2A5 & 2.17 & $1.16-4.05$ & 0.01 & 1.84 & $0.88-3.87$ & 0.1 \\
\hline GSS & 0.46 & $0.24-0.86$ & 0.01 & 0.65 & $0.32-1.31$ & 0.22 \\
\hline$L D H B$ & 0.42 & $0.21-0.85$ & 0.01 & 0.61 & $0.28-1.28$ & 0.19 \\
\hline PANK1 & 0.42 & $0.21-0.86$ & 0.01 & 0.43 & $0.19-0.98$ & 0.04 \\
\hline$I D H 3 G$ & 0.25 & $0.07-0.84$ & 0.02 & 0.34 & $0.11-1.16$ & 0.08 \\
\hline$S C D$ & 2.31 & $1.1-4.87$ & 0.02 & 1.56 & $0.72-3.38$ & 0.25 \\
\hline SDHA & 0.42 & $0.18-0.98$ & 0.04 & 0.51 & $0.21-1.17$ & 0.11 \\
\hline
\end{tabular}

Table 2. Genes associated with overall survival in Cox Proportional Hazard Model. IC95\% confidence interval of $95 \%$. Genes highlighted in bold were independently associated with overall survival and selected to Molecular Based Score. ${ }^{1}$ Hazard ratios (HRs) $>1$ or $<1$ indicate that higher or lower gene expression predicts increased risk of death, respectively.

Internal validation. Based on the unique characteristics of this cohort, mainly by microarray-based transcriptomic data from CD34+ cells, we decided to internally validate our data using the bootstrap resampling procedure. The bootstrap results are depicted in Table 5, and, for all time-points, the procedure yielded a mean $95 \% \mathrm{CI}$ virtually identical to its original match. In addition, the pairwise hypothesis test showed a strong significance $(P<0.001)$ for the difference across the distributions' means for all comparisons. The procedure showed the stability of MBS prediction for 2- and 3-years OS and reinforce the validity of its prediction in a new, but similar, patient collective.

Molecular-Based Score categories are associated with differential gene expression signatures. To further understand the potential mechanisms by which MBS entities regulate hematopoietic progenitor-associated transcriptional programs, we comprehensively compared the transcriptomics signatures among MBS risk categories. Gene set enrichment analysis (GSEA) revealed that increasing MBS risk (i.e. favourable versus ( $v s$ ) intermediate; favourable $v s$ adverse; and intermediate $v s$ adverse) was consistently characterized by upregulation of genes related to oxidative phosphorylation, upregulation of controllers circuits of the cell cycle progression (e.g. G2M_checkpoint and E2F_Targets), and fatty-acid metabolism (Fig. 4A-C; Supplemental Table 1). For specific comparisons, favourable MBS patients were positively enriched with a transcriptional program of megakaryocytic-erythroid progenitor $(\mathrm{MEP})^{16}$ and negative enrichment with leukemic stem cell signature ${ }^{17}$ compared with adverse patients (Fig. 4D). In accordance with the previous observations, favourable patients presented a positive enrichment with mitochondria metabolism ${ }^{18}$ and downregulated genes in hematopoietic stem cell ${ }^{19}$ (Fig. 4E). Adverse MBS patients presented negative enrichment with MEP and downregulated genes in leukemic stem cell (Fig. $4 \mathrm{~F}$ ).

Applying stringent statistical criteria (upregulation: $\log 2$ fold change $>1.5$; downregulation $<-1.5$, all $P<0.05$ ), we identified differentially expressed genes (DEG) for the following comparisons: favourable vs intermediate ( 8 upregulated and 16 downregulated), favourable vs adverse (10 upregulated and 129 downregulated) and intermediate vs adverse (5 upregulated and 42 downregulated) (Fig. 4G-I). Unsupervised hierarchical clustering of transcriptomic data clearly segregated favourable and adverse patients with distinctive DEG signature (Fig. 4J). Taken together, these results suggest that MBS risk categories can efficiently stratify differential transcriptional programs, especially related to cellular energetics and hematopoietic progenitor differentiation.

\section{Discussion}

Here, we described a new prognostic scoring system for patients with MDS based on gene-expression of five metabolic enzymes in CD34+ cells, useful to distinguish patients at three risk categories. Regardless of the wide clinical application of IPSS- $\mathrm{R}^{20}$ for risk assessment in MDS, refining its prognostic function with additional clinical information ${ }^{21}$, flow-cytometr ${ }^{22}$, or mutations ${ }^{23}$ has been of great interest, whereas gene expression analysis it has been underexplored for this purpose. Our proposed MBS efficiently discriminate very-low and low IPSS-R in three risk categories, as well as identified a subset of very favourable prognosis among non-low IPSS-R patients. As far as we know, only two gene expression-based risk scores were published for MDS patients ${ }^{4,5}$, and because both of them have used bulk of bone marrow mononuclear cells, its translation to MDS biology is limited. We 
A

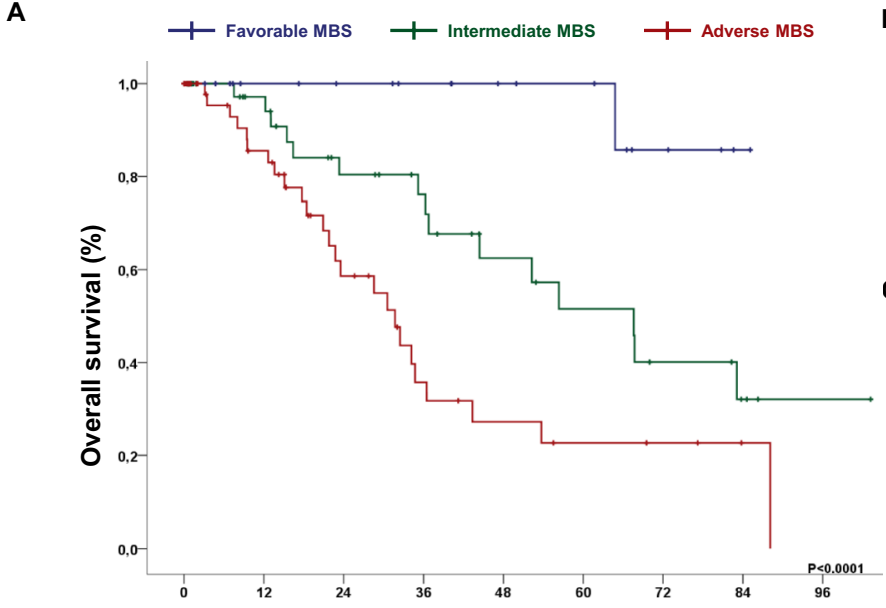

B

Risk Group Score $\quad$ N (\%) Median OS 3-years OS (95\% $\quad$ Cl)

\begin{tabular}{|lcccc|}
\hline Favorable & 0 & $22(18 \%)$ & Not reached & $100 \%(100-100 \%)$ \\
\hline Intermediate & 1 & $46(38 \%)$ & 67.6 months & $76 \%(62-93 \%)$ \\
\hline Adverse & $\geq 2$ & $53(44 \%)$ & 31.7 months & $35 \%(22 \%-59 \%)$ \\
\hline
\end{tabular}

C

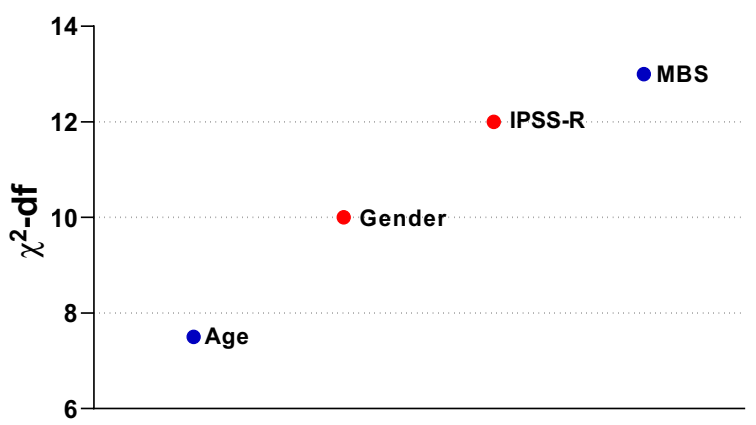

D

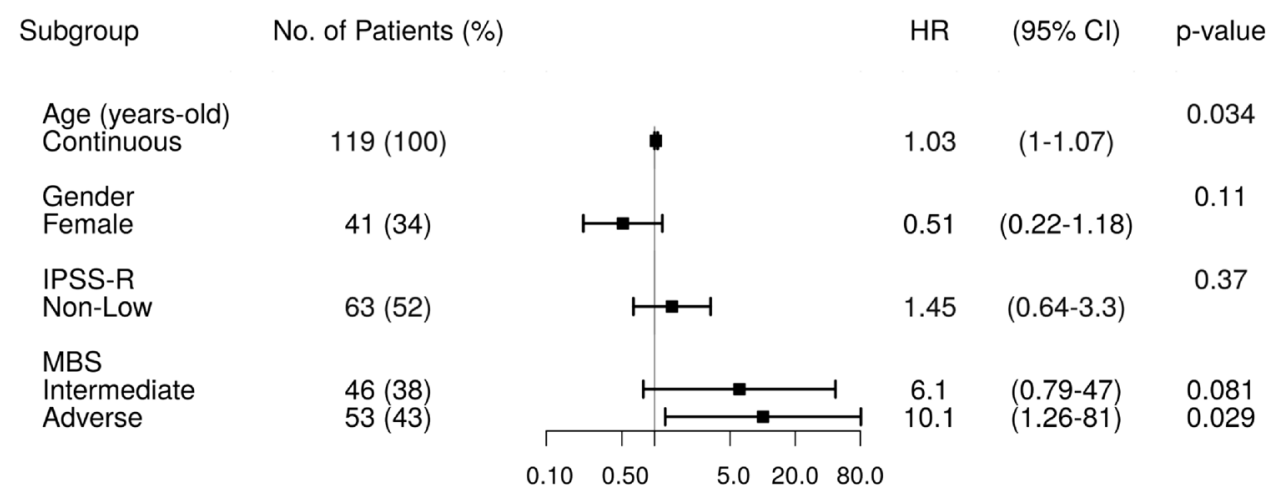

Figure 2. Survival analyses of Molecular-Based Score (MBS) on overall survival (OS) of myelodysplastic syndrome. (A) Kaplan-Meier curves for the three MBS risk categories. (B) MBS was built based on gene expression of ACLY, ANPEP, PANK1, PKM and SLC25A5. MBS efficiently identify three risk groups. (C) Significance $\left(\chi^{2}\right.$-statistic) of each covariate for prediction of OS in the multivariate model, in which higher values represents increased predictive capacity; df: degrees of freedom. (D) Forest plot for multivariable analysis identified adverse risk-MBS and age as independent predictors of OS. Hazard ratios (HR) $>1$ indicates that increasing values for continuous variable or the first factor for categorical variables has the poorer outcome. HR and their respective $95 \%$ confidence interval $(95 \% \mathrm{CI})$ are indicated with black square and a line, respectively. IPSS-R non-low patients included intermediate, high and very-high patients.

have demonstrated that deregulated gene expression in at least two of selected genes is capable to independently predict poorer OS in MDS, with superior prediction capacity than IPSS-R.

The high degree of molecular complexity in MDS represents a challenge to properly define the contribution of all alterations to the pathophysiology of these diseases. Moreover, the majority of MDS biomarkers is still based on mutational profiling ${ }^{24,25}$. Despite the limitation in implementing molecular investigations in clinical setting, particularly in low- and middle-income countries, several initiatives had efficiently established molecular tests validated for risk assessment for other myeloid neoplasms ${ }^{26,27}$.

The strong prognostic function of the MBS across the spectrum of MDS entities and risk categories indicates that perturbations caused by driver molecular alterations might result in metabolic reprogramming and that the MBS is capable to efficiently capture these downstream consequences. Based on Molecular-Based Score classification, we were able to identify patients with differential transcriptional programs that reflect an increased mitochondrial respiration capacity, protein synthesis and, molecular signature related to more mature hematopoietic progenitors in MBS favourable- and intermediate-risk comparing with adverse-risk. Stemness-related transcriptional signature is recognized as a relevant predictor of inferior survival in acute myeloid leukaemia ${ }^{26}$. Moreover, more mature hematopoietic progenitors, such as multipotent and myeloid progenitors, show increased baseline oxygen consumption, mitochondrial ATP production, and respiratory capacity than HSC ${ }^{28}$. Therefore, is conceivable that high $\mathrm{MBS}$ risk patients have $\mathrm{CD} 34^{+}$cells in a more undifferentiated state, related to its reduced 


\begin{tabular}{|c|c|c|c|c|c|c|c|c|c|c|c|c|c|}
\hline \multirow[b]{3}{*}{ Characteristics } & \multirow{2}{*}{\multicolumn{3}{|c|}{ All patients }} & \multicolumn{9}{|c|}{ Molecular-Based Score } & \multirow[b]{3}{*}{$P$ value } \\
\hline & & & & \multicolumn{3}{|c|}{ Favorable risk } & \multicolumn{3}{|c|}{ Intermediate risk } & \multicolumn{3}{|c|}{ Adverse risk } & \\
\hline & No & $\%$ & Median (range) & No & $\%$ & Median (range) & No & $\%$ & Median (range) & No & $\%$ & Median (range) & \\
\hline $\mathrm{N}$ & 159 & 100 & & 28 & 17.6 & & 60 & 37.8 & & 71 & 44,6 & & \\
\hline Gender & & & & & & & & & & & & & 0.08 \\
\hline Female & 57 & 35.8 & & 15 & 46.4 & & 21 & 35 & & 21 & 30 & & \\
\hline Male & 102 & 64.2 & & 13 & 53.6 & & 39 & 65 & & 50 & 70 & & \\
\hline Age, years & & & $67(19-87)$ & & & $63(32-82)$ & & & $67(19-87)$ & & & $67(33-87)$ & 0.439 \\
\hline Bone marrow blasts, \% & & & $4(0-63)$ & & & $2.5(0-14)$ & & & $3(0-63)$ & & & $8.5(0-46)$ & $<0.001$ \\
\hline Hemoglobin, $\mathrm{g} / \mathrm{dL}$ & & & $9.5(4.5-14.6)$ & & & $10(6.9-11.9)$ & & & $9.45(5.4-14.6)$ & & & $9.8(4.5-14.4)$ & 0.952 \\
\hline $\begin{array}{l}\text { Absolute neutrophil } \\
\text { count }, \times 10^{3} / \mu \mathrm{L}\end{array}$ & & & $1.8(0.08-15.2)$ & & & $2.5(0.91-5.36)$ & & & $2.3(0.38-6.4)$ & & & $1.3(0.08-15.2)$ & 0.003 \\
\hline Platelets, $\times 10^{3} / \mu \mathrm{L}$ & & & $152(10-1042)$ & & & $250(38-787)$ & & & $157(16-604)$ & & & $109(10-1042)$ & 0.007 \\
\hline Transfusion dependency & & & & & & & & & & & & & 0.627 \\
\hline Yes & 58 & 44.3 & & 9 & 57.1 & & 27 & 55.1 & & 30 & 52.7 & & \\
\hline No & 73 & 55.7 & & 16 & 32.1 & & 22 & 44.9 & & 27 & 47.3 & & \\
\hline IPSS-R & & & & & & & & & & & & & $<0.001$ \\
\hline Very-low & 27 & 17.0 & & 8 & 28.6 & & 14 & 23.3 & & 5 & 7.1 & & \\
\hline Low & 53 & 33.3 & & 12 & 42.9 & & 26 & 43.3 & & 15 & 21.4 & & \\
\hline Intermediate & 44 & 27.7 & & 5 & 17.9 & & 15 & 25 & & 24 & 33.8 & & \\
\hline High & 23 & 14.5 & & 3 & 10.7 & & 1 & 1.7 & & 19 & 26.8 & & \\
\hline Very-high & 12 & 7.5 & & 0 & 0 & & 4 & 6.7 & & 8 & 11.3 & & \\
\hline WHO 2008 category & & & & & & & & & & & & & $<0.001$ \\
\hline RA & 13 & 8.2 & & 1 & 3.6 & & 9 & 15 & & 3 & 4.2 & & \\
\hline RCMD & 27 & 17.0 & & 1 & 3.6 & & 16 & 26.7 & & 10 & 14.1 & & \\
\hline RCMD-RS & 22 & 13.8 & & 7 & 25 & & 10 & 16.7 & & 5 & 7 & & \\
\hline RARS & 14 & 8.8 & & 8 & 28.6 & & 4 & 6.7 & & 2 & 2.9 & & \\
\hline RARS-T & 6 & 3.8 & & 4 & 14.3 & & 2 & 3.3 & & 0 & 0 & & \\
\hline MDS with 5q- & 6 & 3.8 & & 2 & 7.1 & & 4 & 6.7 & & 0 & 0 & & \\
\hline RAEB & 28 & 17.6 & & 1 & 3.6 & & 9 & 15 & & 18 & 25.3 & & \\
\hline RAEB-2 & 28 & 17.6 & & 4 & 14.3 & & 3 & 5 & & 21 & 29.6 & & \\
\hline AML-MDS & 7 & 4.4 & & 0 & 0 & & 1 & 1.7 & & 6 & 8.4 & & \\
\hline NA & 8 & 5.0 & & 0 & 0 & & 2 & 3.3 & & 6 & 8.4 & & \\
\hline \multicolumn{14}{|l|}{ Mutations } \\
\hline$S F 3 B 1$ & 37 & 29.8 & & 14 & 50 & & 15 & 31.9 & & 8 & 14.8 & & $<0.001$ \\
\hline TET2 & 33 & 20.8 & & 5 & 21.7 & & 14 & 29.8 & & 14 & 25.6 & & 0.765 \\
\hline$A S X L 1$ & 21 & 13.2 & & 2 & 8.7 & & 6 & 12.8 & & 13 & 24.1 & & 0.161 \\
\hline SRSF2 & 16 & 10.1 & & 1 & 4.3 & & 6 & 12.7 & & 9 & 16.7 & & 0.336 \\
\hline DNMT3A & 13 & 8.2 & & 3 & 13 & & 7 & 14.9 & & 3 & 5.5 & & 0.282 \\
\hline RUNX1 & 8 & 6.5 & & 0 & 0 & & 1 & 2.1 & & 7 & 12.9 & & 0.03 \\
\hline$U 2 A F 1$ & 8 & 6.5 & & 1 & 4.3 & & 1 & 2.1 & & 6 & 11.2 & & 0.168 \\
\hline
\end{tabular}

Table 3. Baseline characteristics of patients included for Molecular-Based Score. IPSS-R: Revised International Prognostic Score System; RA: refractory anemia; RCMD: refractory cytopenia with multilineage dysplasia; RCMD-RS: refractory cytopenia with multilineage dysplasia with ring sideroblasts; RARS: refractory anemia with ring sideroblasts; RARS-T: refractory anemia with ring sideroblasts and thrombocytosis; RAEB: refractory anemia with excess blasts. AML-MDS: acute myeloid leukemia with myelodysplastic alterations; NA: Not available.

mitochondrial respiration capacity and cell cycle progression. As a consequence, this delayed haematopoiesis could result in more severe cytopenia in peripheral blood and accumulation of blasts in the bone marrow.

Using advanced stage MDS patients, it has already been demonstrated that $\mathrm{CD} 34^{+} \mathrm{CD} 123^{+}$primitive stem cell is responsible for clonal maintenance and expansion. This compartment has distinctive metabolic characteristics, with activation of protein synthesis machinery and increased oxidative phosphorylation, in comparison to $\mathrm{CD} 34^{+} \mathrm{CD} 123^{-}$counterparts ${ }^{13}$. Conversely, in our study, we demonstrated that lower MBS risk was associated with increased oxidative phosphorylation and protein biosynthesis signatures. We may hypothesize that metabolic reprogramming in $\mathrm{CD} 123^{+}$cells occurs to a different extent for non-advanced stage MDS patients. Indeed, the IL3RA is not differentially expressed among MBS risk categories (Supplemental Fig. 2). As we used transcriptomic from CD34+ bulk cells, the molecular signatures that we observed are probably related to other more frequent subsets of cells. In addition, ectopic expression of SF3B1 mutations in breast cells was associated 


\begin{tabular}{|l|c|c|c|l|}
\hline Factors & 2-years OS (95\%CI) & 3-years OS (95\%CI) & P value $^{1}$ & AUC \\
\hline IPSS-R & AU \\
\hline Very-low & $78 \%(59-100 \%)$ & $78 \%(59-100 \%)$ & 0.004 & $0.57(0.46-0.67)$ \\
\hline Low & $85 \%(73-98 \%)$ & $74 \%(60-92 \%)$ & & \\
\hline Intermediate & $69 \%(50-93 \%)$ & $43 \%(23-80 \%)$ & & \\
\hline High & $69 \%(44-100 \%)$ & $55 \%(30-100 \%)$ & & \\
\hline Very-high & $43 \%(18-100 \%)$ & $43 \%(18-100 \%)$ & & \\
\hline MBS & & \\
\hline Favourable & $100 \%(100-100 \%)$ & $100 \%(100-100 \%)$ & $<0.001$ & $0.70(0.62-0.78)$ \\
\hline Intermediate & $80 \%(67-96 \%)$ & $76 \%(62-93 \%)$ & & \\
\hline Adverse & $59 \%(44-77 \%)$ & $35 \%(22-59 \%)$ & & \\
\hline
\end{tabular}

Table 4. Overall survival for IPSS-R and molecular based score (MBS). IC95\% confidence interval of 95\%; IPSS-R International Prognostic Score System-Revised. ${ }^{1}$ Log-rank test.
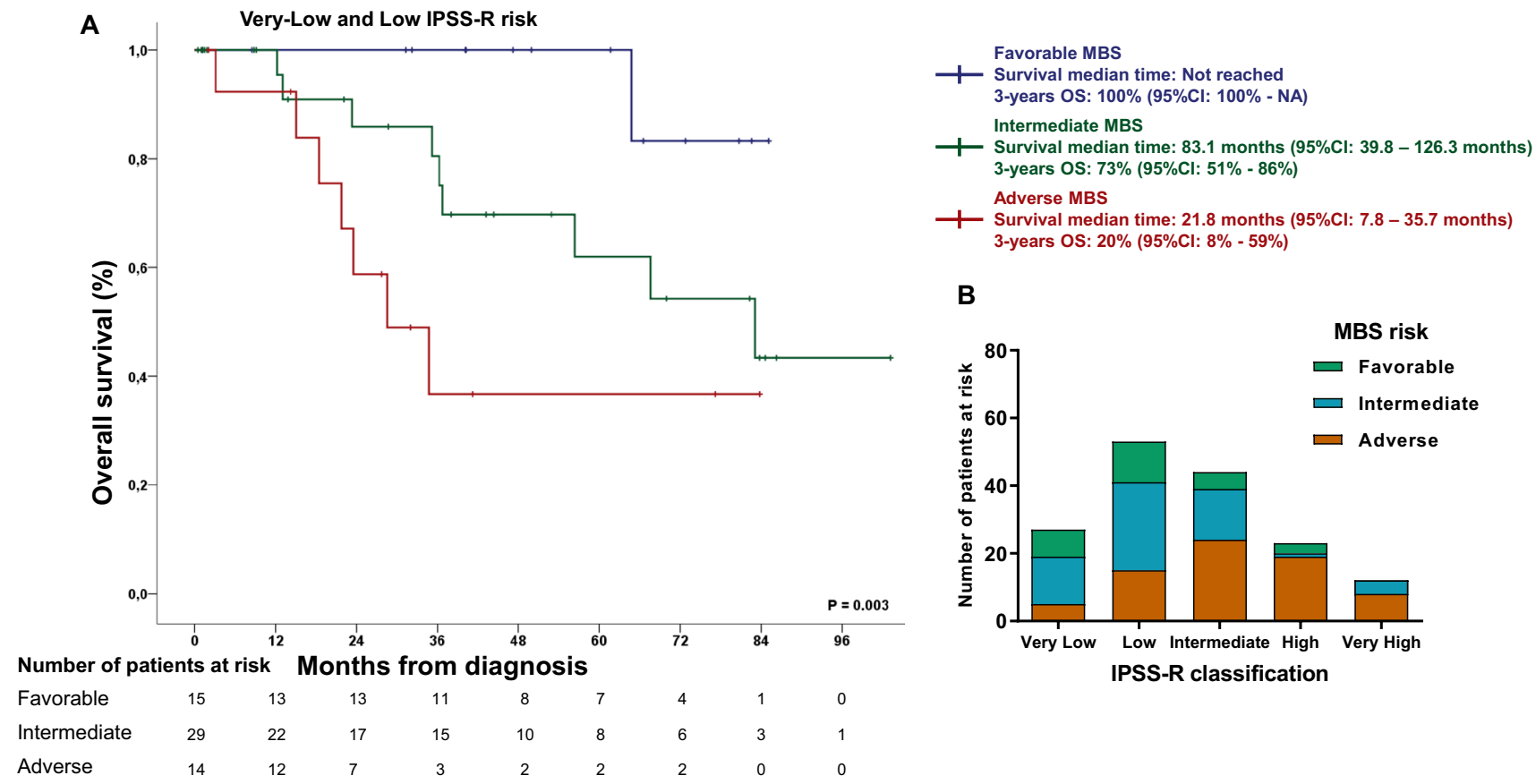

B

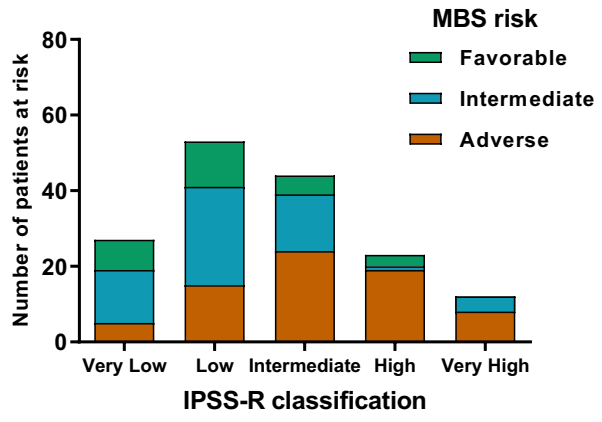

Figure 3. Molecular-Based Score (MBS) prognostic prediction in IPSS-R very-low- and low-risk patients, and distribution across all IPSS-R risk categories. (A) Kaplan-Meier curves of MBS on overall survival (OS) of IPSS-R very-low and low-risk myelodysplastic syndrome. (B) Distribution of MBS across all IPSS-R classification.

\begin{tabular}{|l|c|c|c|c|}
\hline MBS & 2-years OS (95\%CI) & $\boldsymbol{P}_{\text {value }}^{\mathbf{1}}$ & 3-years OS (95\%CI) & $\boldsymbol{P}_{\text {value }^{\mathbf{1}}}$ \\
\hline Favourable & $100 \%(100-100 \%)$ & $<0.001$ & $100 \%(100-100 \%)$ & $<0.001$ \\
\hline Intermediate & $81 \%(62-93 \%)$ & & $76 \%(57-90 \%)$ & \\
\hline Adverse & $58 \%(42-75 \%)$ & & $35 \%(19-56 \%)$ & \\
\hline
\end{tabular}

Table 5. Bootstrap $(\mathrm{R}=1000)$ for 2 -years and 3 -years OS. ${ }^{1}$ Kruskal-Wallis Test.

with disrupted mitochondrial respiration capacity ${ }^{14}$. SF3B1 mutated MDS is considered as having a good prognosis and was recently proposed as a specific disease subtype ${ }^{29}$. Favourable MBS-risk was associated with SF3B1 mutation (Table 3) and as having an oxidative phosphorylation signature. Then, we propose that disruption of mitochondrial complex III mediated by mutant SF3B1 could be dependent on the cellular context, and the metabolic consequences of SF3B1 mutations in CD34 ${ }^{+}$of MDS patients still of major importance.

Ideally, validation of a new prognostic model should determine its capacity in a new data-set scenario. However, external validation is not feasible in most situations. The cohort used in this manuscript shows some unique 

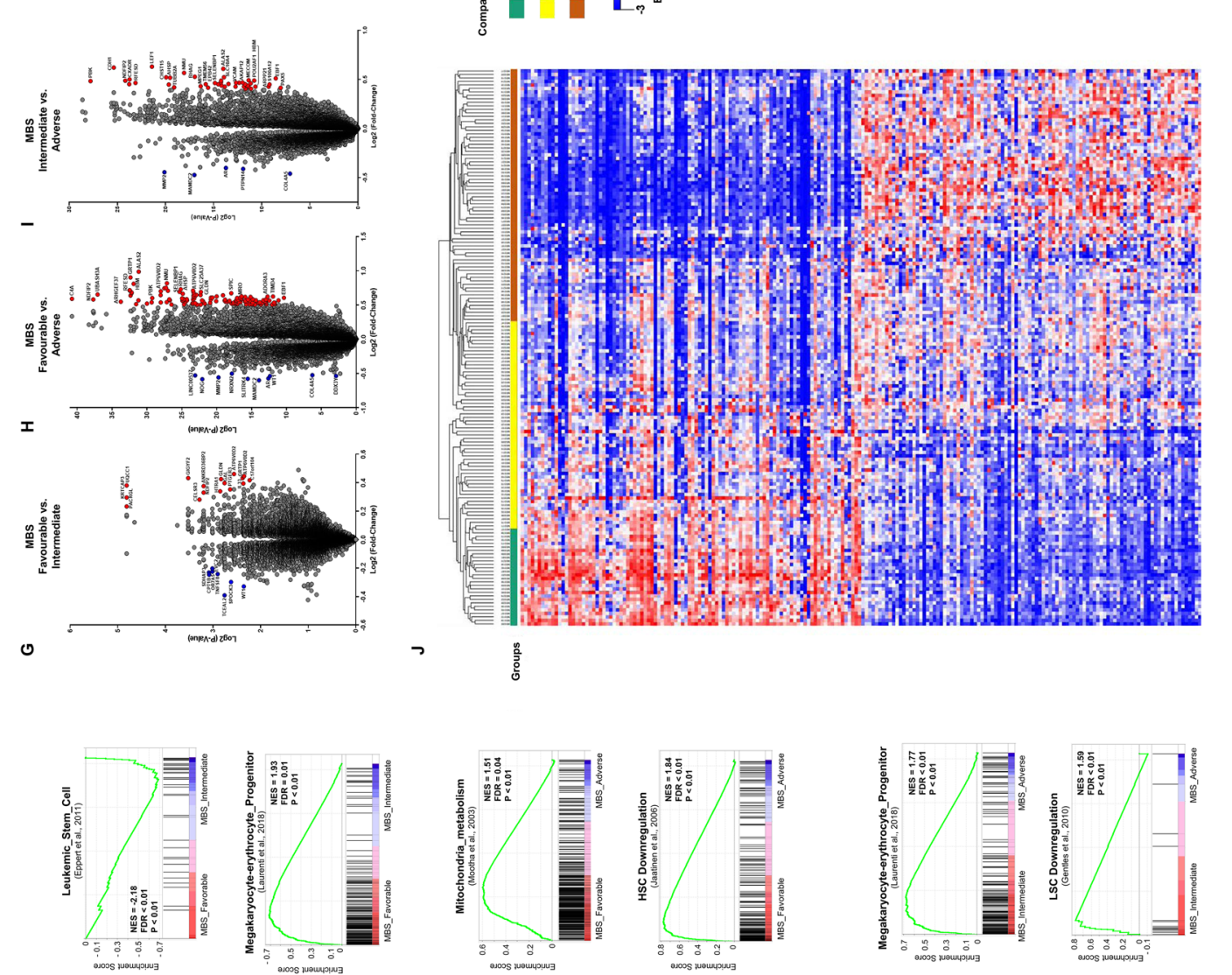

Hin

口

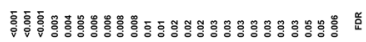

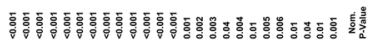

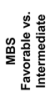
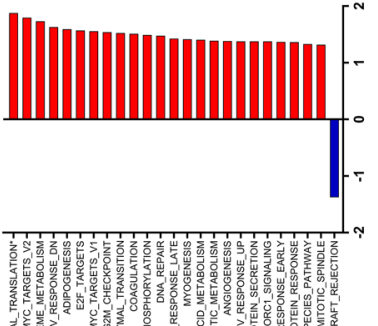

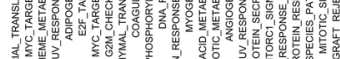

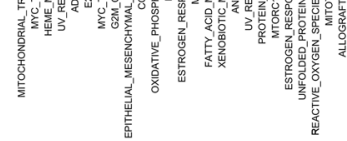

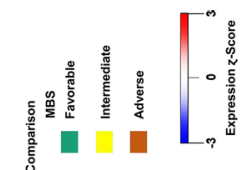
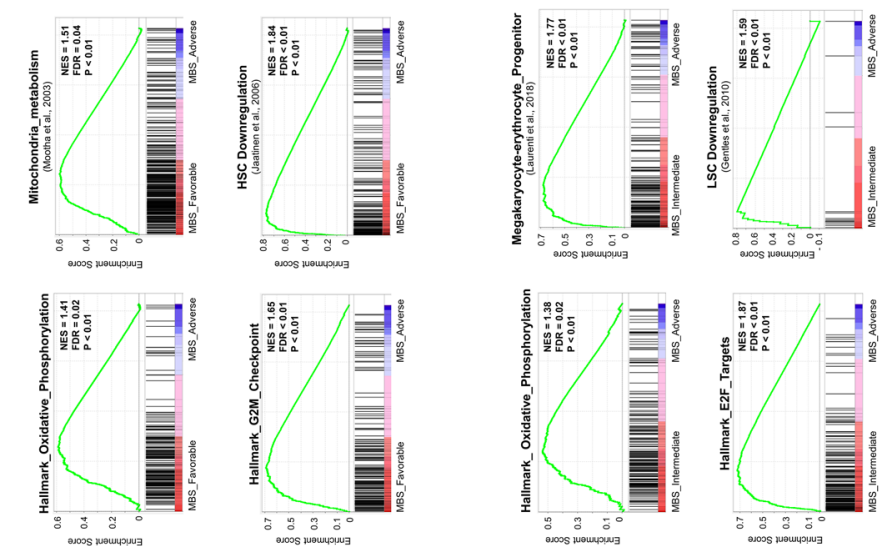

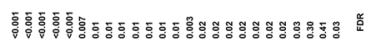

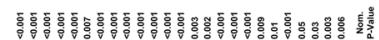
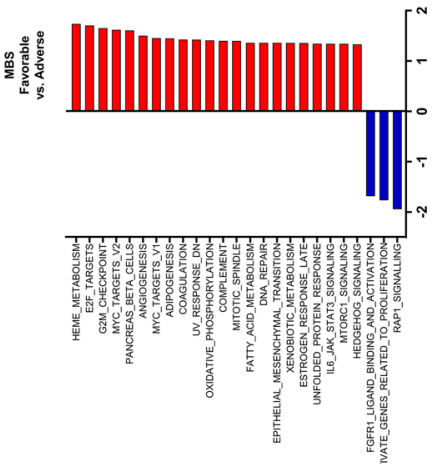

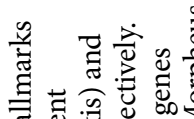

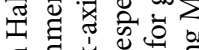

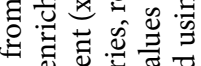

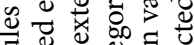

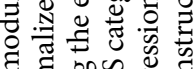

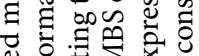

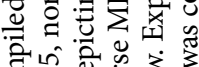

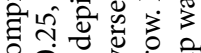

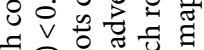

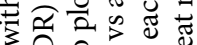

安氙芯芯

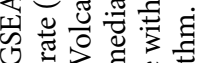

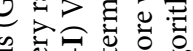

की

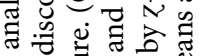

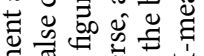

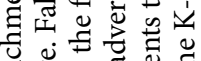

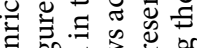

屯

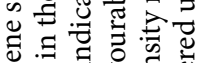

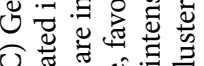

1 तु

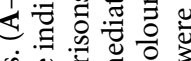

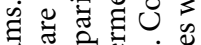

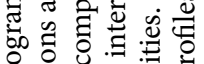

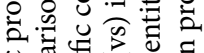

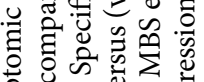

औै के क्षे

ज्ञ

ส

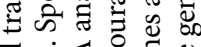

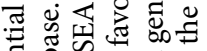

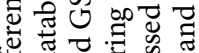

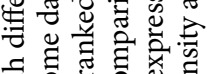

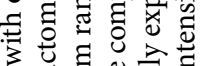

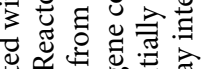

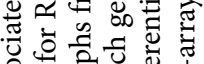

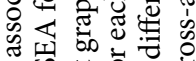

氙苞泀 8

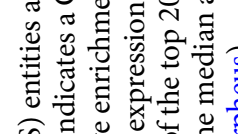

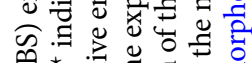

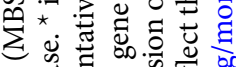

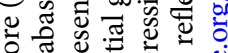

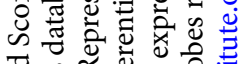

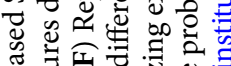

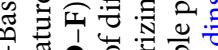

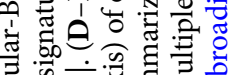

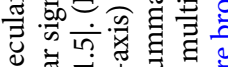

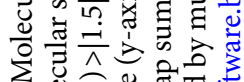

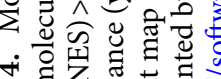

-

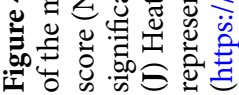


characteristics, such as: 1) transcriptomic data from microarray of CD34 ${ }^{+}$cells, 2) and availability of clinical and demographic data, such as survival, gender, haematological parameters and risk stratification, as well as mutation data. To overcome the impossibility of external validation, we considered internal validation using bootstrap resampling method to evaluate both predictive accuracy and to check overfitting. Of note, this procedure is aligned with the best analytical rigor and was widely used in clinical studies with singular characteristics ${ }^{30-33}$. Independent external cohorts' validations and evaluations in the context of response to different therapies would reinforce the clinical relevance of the proposed score.

The proposition of more efficient and less toxic new therapies is dependent on the ability to exploit a specific weakness that is inherited preferentially in the neoplastic stem cell population. The identification of the MBS for MDS patients contributes to the knowledge of disease pathobiology and provides novelty data according to altered cellular metabolism of the MDS-initiating cell.

\section{Methods}

Clinical and molecular data. Patients' features, mutational status and CD $34^{+}$cells transcriptome data from 159 MDS patients and 17 healthy donors are publicly available at Gene Expression Omnibus (GEO-NCBI; GSE58831 $)^{34}$. Briefly, classification of MDS was updated at sample collection and made according to World Health Organization criteria ${ }^{35}$, while risk stratification determined by IPSS- $\mathrm{R}^{20}$. All patients and healthy controls were from Europe and the centres included: Oxford and Bournemouth (UK), Duisburg (Germany), Stockholm (Sweden) and Pavia (Italy). Baseline features for entire cohort are included in Table 3.

Expression of 37 genes that codify to enzymes related to glycolysis, mitochondrial tricarboxylic acid cycle and oxidative phosphorylation transcriptionally regulated and previously listed as a phenotypic modifiers across different cancer types ${ }^{36-38}$ were selected to interrogate its differential gene expression and predictive outcome function (Table 1).

Transcriptomic analysis. Diagnosis $\mathrm{CD}^{+} 4^{+}$cells were enriched from mononuclear cells using CD34 MicroBeads (Miltenyi Biotec, Germany). For each sample, total RNA was extracted using TRIZOL (Invitrogen, UK) and 50 ng were amplified and labelled using Two-Cycle cDNA Synthesis and the Two-Cycle Target Labelling and Control Reagent kits (Affymetrix, USA). Ten $\mu \mathrm{g}$ of cRNA was hybridized to Affymetrix GeneChip Human Genome U133 Plus 2.0 arrays (Affymetrix, USA), covering 47000 transcripts. Normalized gene expression was calculated using a multichip analysis approach ${ }^{39}$. Mutation data were obtained by targeted gene sequencing, using Illumina Platform, designed to cover 111 genes implicated in myeloid neoplasms pathobiology ${ }^{40}$.

The quantile normalized gene expression was used for a ranking using limma-voom package at Galaxy (https ://usegalaxy.org/) comparing MBS groups (i.e. favourable versus intermediate, favourable versus adverse, and intermediate versus adverse). Pre ranked gene set enrichment analysis (GSEA) was performed using GSEA 4.0.3 software $^{41}$. The gene sets curated by MSigDB hallmark, reactome, hematopoietic progenitors, mitochondrial, and apoptosis were selected for comparisons. Volcano plots computing differentially expressed across MBS entities were constructed correlating the $\log _{2}$-adjusted $P$ value and $\log _{2}$-Fold-Change in GraphPad Prism 8.0 (GraphPad Software, USA). Heat map was constructed to represent top differentially expressed genes in MBS risk groups using the online available tool Morpheus (https://software.broadinstitute.org/morpheus).

Statistical considerations. Descriptive analyses were performed for patient baseline features. Fisher's exact test or Chi-square test, as appropriate, was used to compare categorical variables. Non-parametric MannWhitney test was used to compare continuous variables.

In order to optimize the cut off selection for gene expression, we opted to use "cutpointr" package and automatically determined the critical points for each 37 genes using receiver operating characteristic curve analysis ${ }^{42}$ and the $\mathrm{C}$-index ${ }^{43}$ pre-selected for our score (Table 1 ). After dichotomization, we evaluated the predictive capacity of each gene (Table 2) in a univariate and multivariate way by Proportional Hazard Cox regression analysis using the "Cox_HR" function of "SurvivalAnalysis" package ${ }^{44,45}$. Genes $(\mathrm{n}=11)$ significantly associated with survival in univariate analysis were individually considered in multivariate analysis using age, gender, and IPSS-R stratification as cofounders. Five genes independently predicted OS and were selected for MBS estimation.

MBS was calculated by computing 1 for every molecular risk factor, e.g. high expression of $A N P E P$ and $P K M$, and low expression of $A C L Y, P A N K 1$ and SLC25A5, varying from 0 (summing zero molecular risk factor) to 5 (summing all five molecular risk factor). MBS risk groups were determined by Kaplan-Meyer inspection ${ }^{46}$, and were defined as MBS-Favourable for patients without molecular risk factor, MBS-Intermediate for patients with one molecular risk factor and as MBS-Adverse with two or more molecular risk factors.

To determine the predictive capacity for MBS, a receiver operating characteristic (ROC) curve and the respective concordance statistics (C-statistics) were performed. The respective area under the curve (AUC) were derived from an $\mathrm{R}$ implementation of DeLong's algorithm ${ }^{47}$. To determine if MBS predictive capacity is superior to IPSS-R, we calculated differences between $\mathrm{AUC}(\Delta-\mathrm{AUC})$ as $\Delta-\mathrm{AUC}=\mathrm{AUC}_{\mathrm{MBS}}-\mathrm{AUC}_{\mathrm{IPSS}-\mathrm{R}}$. For this purpose, we performed 10,000 bootstrap resampling procedure and calculated the $\Delta$-AUC for each interaction. Positive values represent that MBS performed better than IPSS- $\mathrm{R}^{48}$.

The bootstrap resampling procedure performed 1,000 resampling of the original cohort and calculated all clinical endpoints in two different time points (2-year, and 3-year) for three MBS-categories (favourable-, intermediate- and adverse-risk MBS). The procedure also estimated their respective $95 \%$ confidence interval (CI) computing the bias-corrected and accelerated bootstrap interval.

Proportional hazards (PH) assumption for each continuous variable of interest was tested. Linearity assumption for all continuous variables was examined in logistic and $\mathrm{PH}$ models using restricted cubic spline estimates of the relationship between the continuous variable and log relative hazard/risk. All $P$ values were two sided 
with a significance level of 0.05 . All calculations were performed using Stata Statistic/Data Analysis version 12 (Stata Corporation, USA), Statistical Package for Social Sciences 19 (SPSS 19) and R 3.5.2 (The CRAN project, www.r-project.org) software.

Received: 2 July 2020; Accepted: 29 December 2020

Published online: 18 January 2021

\section{References}

1. Swerdlow, S. H. et al. World Health Organization Classification of Tumours of Haematopoietic and Lymphoid Tissues. (International Agency for Research on Cancer (IARC), 2017).

2. Bejar, R., Levine, R. \& Ebert, B. L. Unraveling the molecular pathophysiology of myelodysplastic syndromes. J. Clin. Oncol. 29, 504-515 (2011).

3. Ogawa, S. Genetics of MDS. Blood 133, 1049-1059 (2019).

4. Thol, F. et al. Prognostic significance of combined MN1, ERG, BAALC, and EVI1 (MEBE) expression in patients with myelodysplastic syndromes. Ann. Hematol. 91, 1221-1233 (2012).

5. Yao, C.-Y. et al. A 4-lncRNA scoring system for prognostication of adult myelodysplastic syndromes. Blood Adv. 1, 1505-1516 (2017).

6. Lu, J., Tan, M. \& Cai, Q. The Warburg effect in tumor progression: Mitochondrial oxidative metabolism as an anti-metastasis mechanism. Cancer Lett. 356, 156-164 (2015).

7. Kritikou, E. Metabolism: Warburg effect revisited. Nat. Rev. Cancer 8, 247 (2008).

8. Warburg, O. The metabolism of carcinoma cells 1. J. Cancer Res. 9, 148-163 (1925).

9. Schwartz, L., Supuran, C. \& Alfarouk, K. The Warburg Effect and the Hallmarks of Cancer. Anticancer. Agents Med. Chem. 17, 164-170 (2017).

10. Hanahan, D. \& Weinberg, R. A. Hallmarks of cancer: the next generation. Cell 144, 646-674 (2011).

11. Pavlova, N. N. \& Thompson, C. B. The emerging hallmarks of cancer metabolism. Cell Metab. 23, 27-47 (2016).

12. Lagadinou, E. D. et al. BCL-2 inhibition targets oxidative phosphorylation and selectively eradicates quiescent human leukemia stem cells. Cell Stem Cell 12, 329-341 (2013).

13 Stevens, B. M. et al. Characterization and targeting of malignant stem cells in patients with advanced myelodysplastic syndromes. Nat. Commun. 9, 3694 (2018).

14. Brian Dalton, W. et al. Hotspot SF3B1 mutations induce metabolic reprogramming and vulnerability to serine deprivation. J. Clin. Invest. 129, 4708-4723 (2019).

15. Gerstung, M. et al. Combining gene mutation with gene expression data improves outcome prediction in myelodysplastic syndromes. Nat. Commun. 6, 1-11 (2015).

16. Belluschi, S. et al. Myelo-lymphoid lineage restriction occurs in the human haematopoietic stem cell compartment before lymphoidprimed multipotent progenitors. Nat. Commun. 9, 1-15 (2018).

17. Eppert, K. et al. Stem cell gene expression programs influence clinical outcome in human leukemia. Nat. Med. 17, 1086-1094 (2011).

18. Mootha, V. K. et al. PGC-1 $\alpha$-responsive genes involved in oxidative phosphorylation are coordinatelydownregulated in human diabetes. Nat. Genet. 34, 267-273 (2003).

19. Jaatinen, T. et al. Global gene expression profile of human cord blood-derived CD133 + cells. Stem Cells 24, 631-641 (2006).

20. Greenberg, P. L. et al. Revised international prognostic scoring system for myelodysplastic syndromes. Blood 120, 2454-2465 (2012).

21. Montalban-Bravo, G. \& Garcia-Manero, G. Myelodysplastic syndromes: 2018 update on diagnosis, risk-stratification and management. Am. J. Hematol. 93, 129-147 (2018).

22. Alhan, C. et al. Themyelodysplastic syndromes flow cytometric score: a three-parameter prognostic flow cytometric scoring system. Leukemia 30, 658-665 (2016).

23. Tefferi, A. et al. Targeted next-generation sequencing in myelodysplastic syndromes and prognostic interaction between mutations and IPSS-R. Am. J. Hematol. 92, 1311-1317 (2017).

24. Bejar, R. et al. Clinical effect of point mutations in myelodysplastic syndromes. N. Engl. J. Med. 364, 2496-2506 (2011).

25. Bejar, R. et al. Somatic mutations predict poor outcome in patients with myelodysplastic syndrome after hematopoietic stem-cell transplantation. J. Clin. Oncol. 32, 1-9 (2014).

26. Ng, S. W. K. et al. A 17-gene stemness score for rapid determination of risk in acute leukaemia. Nature 540, 433-437 (2016).

27. Nomdedéu, J. F. et al. Feasibility of the AML profiler (Skyline ${ }^{\mathrm{TM}}$ Array) for patient risk stratification in a multicentre trial: a preliminary comparison with the conventional approach. Hematol. Oncol. 35, 778-788 (2017).

28. de Almeida, M. J., Luchsinger, L. L., Corrigan, D. J., Williams, L. J. \& Snoeck, H. W. Dye-independent methods reveal elevated mitochondrial mass in hematopoietic stem cells. Cell Stem Cell 21, 725-729.e4 (2017).

29. Malcovati, L. et al. SF3B1-mutant MDS as a distinct disease subtype: a proposal from the International Working Group for the Prognosis of MDS. Blood 136, 157-170 (2020).

30. Suthanthiran, M. et al. Urinary-cell mRNA profile and acute cellular rejection in kidney allografts. N. Engl. J. Med. 369, 20-31 (2013).

31. Muller, D. C., Johansson, M. \& Brennan, P. Lung cancer risk prediction model incorporating lung function: development and validation in the UK biobank prospective cohort study. J. Clin. Oncol. 35, 861-869 (2017).

32. Marcus, M. W. \& Field, J. K. Is bootstrapping sufficient for validating a riskmodel for selection of participants for a lung cancer screening program?. J. Clin. Oncol. 35, 818-819 (2017).

33. Yu, D. et al. Development and validation of risk prediction models for cardiovascular mortality in Chinese people initialising peritoneal dialysis: a cohort study. Sci. Rep. 8, 1-7 (2018).

34 Gerstung, M. et al. Combining gene mutation with gene expression data improves outcome prediction in Myelodysplastic Syndromes. Nat. Commun. https://doi.org/10.1038/ncomms6901 (2015).

35. Swerdlow, S. et al. WHO Classification of Tumours of Haematopoietic and Lymphoid Tissues. World Health Organization (2017).

36. Chen, W.-L. et al. A distinct glucose metabolism signature of acute myeloid leukemia with prognostic value. Blood 124, 1645-1654 (2014).

37. Qiu, Y. et al. A distinct metabolic signature of human colorectal cancer with prognostic potential. Clin. Cancer Res. 20, 2136-2146 (2014).

38. Pollyea, D. A. et al. Venetoclax with azacitidine disrupts energy metabolism and targets leukemia stem cells in patients with acute myeloid leukemia. Nat. Med. 24, 1859-1866 (2018).

39. Irizarry, R. A. et al. Exploration, normalization, and summaries of high density oligonucleotide array probe level data. Biostatistics 4, 249-264 (2003) 
40. Papaemmanuil, E. et al. Clinical and biological implications of driver mutations in myelodysplastic syndromes. Blood 122, 36163627 (2013).

41. Subramanian, A. et al. Gene set enrichment analysis: A knowledge-based approach for interpreting genome-wide expression profiles. Proc. Natl. Acad. Sci. USA 102, 15545-15550 (2005).

42. Heagerty, P. J. \& Zheng, Y. Survival model predictive accuracy and ROC curves. Biometrics 61, 92-105 (2005).

43. Harrell, F. E., Califf, R. M., Pryor, D. B., Lee, K. L. \& Rosati, R. A. Evaluating the yield of medical tests. JAMA 247, 2543-2546 (1982).

44. Bradburn, M. J., Clark, T. G., Love, S. B. \& Altman, D. G. Survival Analysis Part II: Multivariate data analysis-an introduction to concepts and methods. Br. J. Cancer 89, 431-436 (2003).

45. Multivariate Survival Analysis. Available at: https://cran.r-project.org/web/packages/survivalAnalysis/vignettes/multivariate.html. (Accessed 5th May 2020)

46 Altman, D. G. Practical statistics for medical research (Chapman and Hall, London, 1991).

47. DeLong, E. R., DeLong, D. M. \& Clarke-Pearson, D. L. Comparing the areas under two or more correlated receiver operating characteristic curves: a nonparametric approach. Biometrics 44, 837 (1988).

48. Silveira, D. R. A. et al. Integrating clinical features with genetic factors enhances survival prediction for adults with acute myeloid leukemia. Blood Adv. 4, 2339-2350 (2020).

\section{Acknowledgments}

Funding for this work was supported by Fundação de Amparo à Pesquisa do Estado de São Paulo (FAPESP Grants \#2016/23191-4, \#2017/19864-6, \#2017/23117-1, and \#2013/08135-2), Conselho Nacional de Desenvolvimento Científico e Tecnológico (CNPq; INCTC 2014/50947-7) and Coordenação de Aperfeiçoamento de Pessoal de Nível Superior (CAPES).

\section{Author contributions}

J.L.C-S conceived and designed the study, analysed, and interpreted data, performed the statistical analyses, and drafted the article. D.R.A.S. performed the bootstrap resampling analyses, reviewed the statistical analysis, and reviewed the paper. D.A.P-M, C.O.A.R, A.R.L-A, E.M.R., J.A.M-N, I.B., V.R. contributed with inputs on overall design of study, participated in the interpretation of manuscript data and reviewed the paper. F.T. was the principal investigator, conceived and designed the study and gave final approval of the submitted version.

\section{Competing interests}

The authors declare no competing interests.

\section{Additional information}

Supplementary Information The online version contains supplementary material available at https://doi. org/10.1038/s41598-020-80918-6.

Correspondence and requests for materials should be addressed to F.T.

Reprints and permissions information is available at www.nature.com/reprints.

Publisher's note Springer Nature remains neutral with regard to jurisdictional claims in published maps and institutional affiliations.

(c) (1) Open Access This article is licensed under a Creative Commons Attribution 4.0 International (c) License, which permits use, sharing, adaptation, distribution and reproduction in any medium or format, as long as you give appropriate credit to the original author(s) and the source, provide a link to the Creative Commons licence, and indicate if changes were made. The images or other third party material in this article are included in the article's Creative Commons licence, unless indicated otherwise in a credit line to the material. If material is not included in the article's Creative Commons licence and your intended use is not permitted by statutory regulation or exceeds the permitted use, you will need to obtain permission directly from the copyright holder. To view a copy of this licence, visit http://creativecommons.org/licenses/by/4.0/.

(C) The Author(s) 2021 